(2) Open Access Full Text Article

\title{
Intravitreal triamcinolone acetonide for rebound phenomenon after high-dose intravenous steroid treatment in Vogt-Koyanagi-Harada disease
}

This article was published in the following Dove Press journal:

Clinical Ophthalmology

3 November 2011

Number of times this article has been viewed

\author{
Ik Soo Byon' \\ Ji Hun Kim' \\ Ji Eun Lee ${ }^{1,2}$ \\ Boo Sup Oum ${ }^{1,2}$ \\ 'Department of Ophthalmology, Pusan \\ National University Hospital; ${ }^{2}$ Medical \\ Institute, School of Medicine, Pusan \\ National University, Busan, Republic \\ of Korea
}

\begin{abstract}
The authors report two cases of rebound phenomenon treated with intravitreal triamcinolone acetonide in Vogt-Koyanagi-Harada (VKH) disease. Patients in the acute phase of VKH disease were treated with high-dose intravenous (IV) methylprednisolone (1 g/day) for 3 days. Serous retinal detachment decreased and visual acuity improved during IV steroid treatment. After switching to oral steroid treatment, choroiditis and visual acuity worsened. An injection of triamcinolone acetonide $(4 \mathrm{mg})$ into the vitreous resulted in gradual resolution of subretinal fluid and improvement of visual acuity. Systemic steroids were tapered to discontinuation without a relapse of inflammation. Adjuvant intravitreal triamcinolone is useful in the management of the rebound phenomenon in VKH disease.
\end{abstract}

Keywords: adjuvant intravitreal steroid, serous retinal detachment, visual acuity, choroiditis

\section{Introduction}

Vogt-Koyanagi-Harada (VKH) disease has a unique natural history that comprises four phases: prodromal, acute uveitic, chronic, and chronic recurrent. Of these phases, the acute phase requires intensive treatment. ${ }^{1}$ Delayed or inadequate control of inflammation at this phase may lead to the recurrent phase, which is difficult to manage. Although many physicians popularly use high-dose intravenous (IV) steroids for 3 days before switching to oral steroids, the optimal management for choroidal inflammation in the acute phase has not been established. The authors report two cases of rebound choroidal inflammation after transitioning from high-dose IV steroids to oral steroids that were managed successfully using adjuvant intravitreal steroid injection.

\section{Case report I}

A 43-year-old woman presented with headache, tinnitus, and decreased visual acuity. She had no history or evidence of penetrating ocular trauma, ocular surgery, or ocular or systemic diseases. Her best-corrected visual acuity was 20/60 in the right eye and $20 / 40$ in the left. The anterior segment had no signs of inflammation and fundus examination revealed bullous serous retinal detachments in both eyes. Multiple pinpoint leakages and late pooling within subretinal fluid (SRF) were detected using fluorescein angiography (FA) (Figure 1A and B). Intra- and SRF with septa was noted by spectral domain optical coherence tomography (SD-OCT) using the Cirrus ${ }^{\mathrm{TM}}$ HD-OCT (Carl Zeiss Meditec AG, Jena, Germany) (Figure 1C). A diagnosis of incomplete VKH disease was determined and treatment with high-dose IV methylprednisolone pulse therapy ( $1 \mathrm{~g} /$ day) was administered for 3 days. During IV steroid treatment, the patient's SRF
Correspondence: Ji Eun Lee

Pusan National University Hospital,

I-I0 Ami-dong, Seo-gu, Busan 602-739,

Republic of Korea

$\mathrm{Tel}+9825 \mathrm{I} 2407957$

Fax +9 82 51 2427341

Email jlee@pusan.ac.kr 

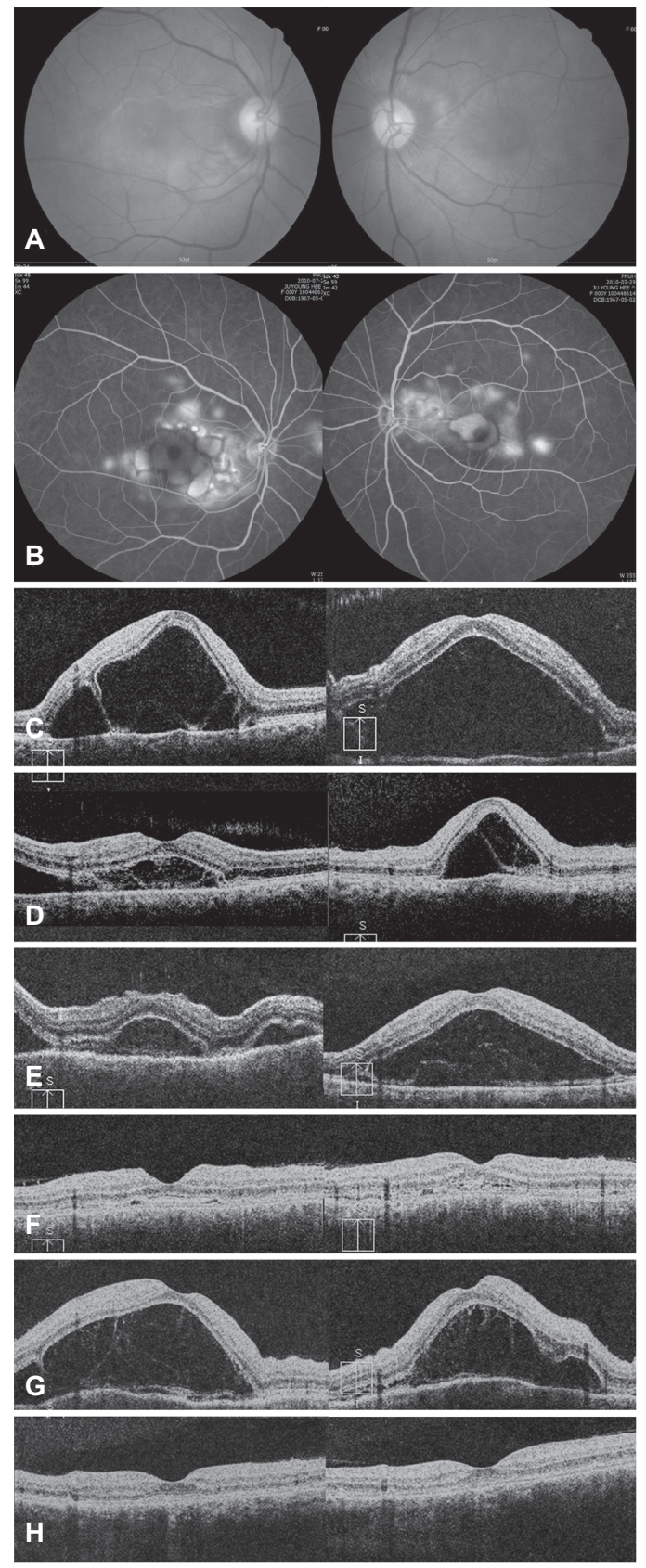

Figure I Case I. (A) Fundus photograph shows bullous retinal detachment in both eyes at presentation. (B) Multiple leakages and poolings in the late phase of fluorescein angiography were detected. (C) Optical coherence tomography image represents intra- and subretinal fluid (SRF) with subretinal septa. (D) SRF was considerably reduced at 3 days after intravenous (IV) pulse steroid therapy. (E) SRF increased at 3 days after changing treatment from IV to oral steroids. (F) SRF resolved at 4 days after IV pulse steroid therapy was restarted. (G) Bullous retinal detachment developed again after changing to oral steroids. (H) SRF resolved after intravitreal injection of triamcinolone acetonide.

decreased and her vision improved to $20 / 40$ in both eyes (Figure 1D). Methylprednisolone therapy was then switched to oral prednisolone $(1 \mathrm{mg} / \mathrm{kg} /$ day $)$. At 3 days after switching, the patient's SRF increased and her vision worsened to 20/160 and 20/60 (Figure 1E). High-dose IV methylprednisolone was then restarted. The patient's SRF reduced and her visual acuity improved remarkably after 4 days (Figure 1F). Systemic steroids were then changed again to an oral formulation. After restarting oral steroid treatment $(1.3 \mathrm{mg} / \mathrm{kg} /$ day $)$, the patient's vision decreased gradually as bullous retinal detachment developed (Figure 1G). While maintaining her oral steroid dose, triamcinolone acetonide $4 \mathrm{mg}$ was injected intravitreally as an adjunctive treatment. The SRF resolved gradually with visual acuity improvement. During a follow-up period of 13 months, intraocular pressure was stable and cataract did not develop. Systemic steroids were tapered to discontinuation without a relapse of inflammation (Figure 1H).

\section{Case report 2}

A 33-year-old man complained of tinnitus and visual disturbance involving the right eye for 2 days. His vision was 20/120 in the right eye and 20/20 in the left. Serous retinal detachment was found in both eyes. FA showed multiple pinpoint leakages and late pooling (Figure 2A and B). SD-OCT detected multilobulated serous detachments (Figure 2C). Evaluations for causes other than VKH disease were negative. The patient was treated for 3 days with high-dose IV methylprednisolone $(1 \mathrm{~g} /$ day $)$ followed by 5 days with oral steroids ( $1 \mathrm{mg} / \mathrm{kg} /$ day). Serous retinal detachment decreased and his vision improved to 20/50 in the right eye during the 3 days while on IV steroid treatment (Figure 2D). After 5 days of oral steroid treatment, the patient's SRF increased and his vision worsened (Figure 2E). Adjuvant triamcinolone acetonide $4 \mathrm{mg}$ was injected into the vitreous of both eyes. Visual acuity and serous detachment improved (Figure 2F). During a follow-up period of 22 months, an elevation of intraocular pressure did not develop and systemic steroids were successfully tapered without a relapse of inflammation.

\section{Discussion}

VKH disease is a disorder of T-cell-mediated autoimmunity directed against melanocytes in the eye, central nervous system, auditory system, and skin. The typical treatment for VKH disease is high-dose systemic steroids, followed by slow tapering over 3-6 months. Although the route of administration of steroids has been reported to have no difference in visual acuity or on the development of visually significant complications, ${ }^{2}$ many physicians prefer an initial therapy of prompt and intensive high-dose IV methylprednisolone over 3 days. It is well known that most patients show a resolution of serous retinal detachment and an improvement of vision within days of receiving IV pulse steroid therapy. ${ }^{3}$

To the authors' knowledge, rebound phenomenon in patients with VKH disease has not been reported before. It may be underestimated because the dosage of oral 


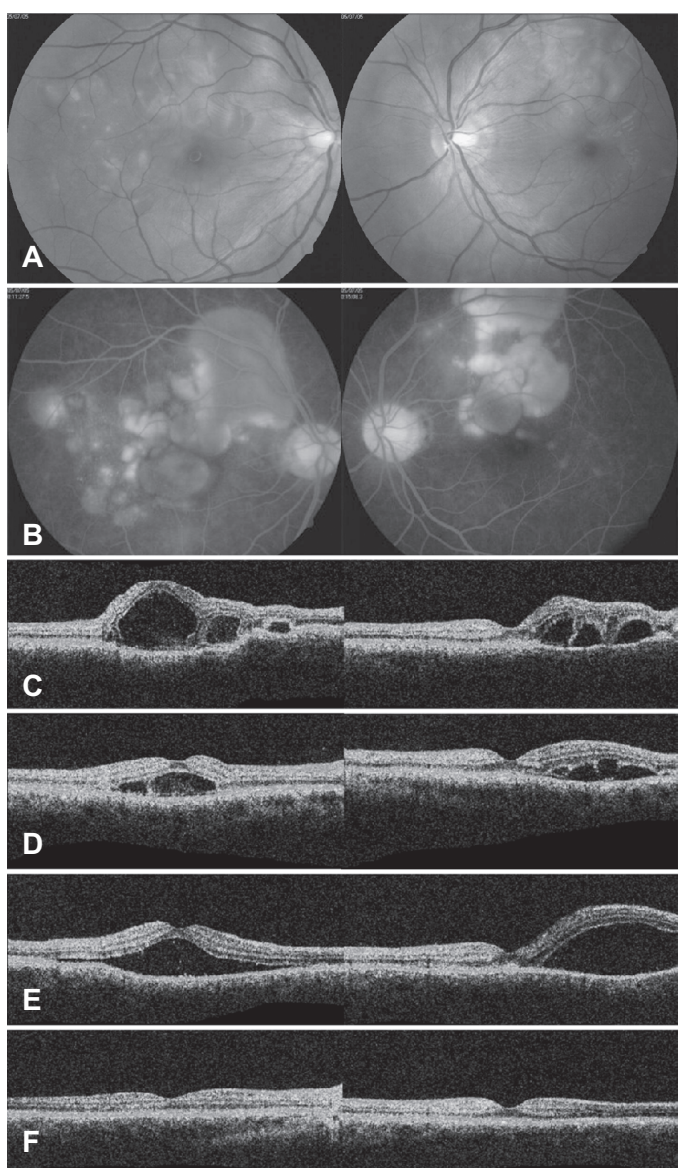

Figure 2 Case 2. (A) Fundus photograph shows serous retinal detachment in both eyes. (B) Multiple leakages and poolings in the late phase of fluorescein angiography were detected. (C) Optical coherence tomography image represents intraretina fluid accumulation and subretinal septa in the multilobular serous detachment at initial visit. (D) Subretinal fluid (SRF) was remarkably reduced at 3 days after highdose intravenous pulse steroid therapy. (E) SRF increased at 5 days after starting oral steroids. (F) After intravitreal injection of adjuvant triamcinolone acetonide, SRF resolved and vision recovered. The patient was successfully tapered off oral steroids without rebound phenomenon.

steroids after transitioning from the IV regimen is similar to the starting dosage in oral steroid monotherapy and the treating physicians do not expect a relapse of inflammation.

However, these two cases presented with relapsing choroiditis after a change in the steroid administration route. It was considered that control of the inflammation was required, because delayed or inadequate control of inflammation may lead to the chronic recurrent phase, which is difficult to manage.
In the first case of rebound phenomenon, choroiditis was relapsed even after the second session of IV pulse steroid therapy. As prolonged systemic treatment with high-dose steroids was expected, intravitreal triamcinolone acetonide was administered to avoid the side effects of systemic steroids. ${ }^{4-6}$ The second case of rebound phenomenon could be managed successfully with adjuvant intravitreal triamcinolone while maintaining the tapering schedule of oral steroids.

\section{Conclusion}

The rebound phenomenon may have been secondary to either an insufficient oral steroid dose or the difference of the dose between IV and oral regimens when transitioning formulations. An intermediate dose may be considered between two formulations to prevent this untoward event. If rebound phenomenon develops, adjuvant intravitreal injection of triamcinolone acetonide is effective in controlling inflammation.

\section{Disclosure}

The authors report no conflicts of interest in this work. The authors did not receive any financial support, and the authors alone are responsible for the content and writing of the paper.

\section{References}

1. Andreoli CM, Foster CS. Vogt-Koyanagi-Harada disease. Int Ophthalmol Clin. 2006;46(2):111-122.

2. Yamanaka E, Ohguro N, Yamamoto S, Nakagawa Y, Imoto Y, Tano Y Evaluation of pulse corticosteroid therapy for Vogt-Koyanagi-Harada disease assessed by optical coherence tomography. Am J Ophthalmol. 2002;134(3):454-456.

3. Read RW, Yu F, Accorinti M, et al. Evaluation of the effect on outcomes of the route of administration of corticosteroids in acute Vogt-KoyanagiHarada disease. Am J Ophthalmol. 2006;142(1):119-124.

4. Karacorlu M, Arf Karakorlu S, Ozdemir H. Intravitreal triamcinolone acetonide in Vogt-Koyanagi-Harada syndrome. Eur J Ophthalmol. 2006;16(3):481-483

5. Andrade RE, Muccioli C, Farah ME, Nussenblatt RB, Belfort R Jr. Intravitreal triamcinolone in the treatment of serous retinal detachment in Vogt-Koyanagi-Harada syndrome. Am J Ophthalmol. 2004;137(3): 572-574.

6. Perente I, Utine CA, Cakir H, Kaya V, Tutkun IT, Yilmaz OF. Management of ocular complications of Vogt-Koyanagi-Harada syndrome. Int Ophthalmol. 2009;29(1):33-37.
Clinical Ophthalmology

\section{Publish your work in this journal}

Clinical Ophthalmology is an international, peer-reviewed journa covering all subspecialties within ophthalmology. Key topics include: Optometry; Visual science; Pharmacology and drug therapy in eye diseases; Basic Sciences; Primary and Secondary eye care; Patien Safety and Quality of Care Improvements. This journal is indexed on
PubMed Central and CAS, and is the official journal of The Society of Clinical Ophthalmology (SCO). The manuscript management system is completely online and includes a very quick and fair peer-review system, which is all easy to use. Visit http://www.dovepress.com/ testimonials.php to read real quotes from published authors. 